ISSN: 1130-3743 - e-ISSN: 2386-5660

DOI: http://dx.doi.org/10.14201/teoredu20162814560

\title{
DE LA IDENTIDAD DEL SER A LA PEDAGOGÍA DE LA DIFERENCIA
}

\author{
From identity of being to pedagogy of difference
}

\section{De l'identité de l'être à la pédagogie de la différence}

\author{
Ignacio Calderón Almendros*, Juan Miguel Calderón Almendros*** \\ y María Teresa RAscón GÓmEz* \\ * Universidad de Málaga. Facultad de Ciencias de la Educación. Departamento \\ de Teoría e Historia de la Educación. Campus de Teatinos, s/n. ica@uma.es; \\ trascon@uma.es \\ ** Departamento de Filosofía. IES Bernardo de Balbuena. Valdepeñas-Ciudad Real. \\ juanmicalderon@gmail.com
}

Fecha de recepción: enero de 2016

Fecha de aceptación: marzo de 2016

\section{RESUMEN}

Las distintas definiciones acerca del ser humano intentan identificar una característica que generalizar para todo el género Homo. Esta cuestión nace del principio de identidad, y tiene fuertes repercusiones en las concepciones antropológicas sobre las que se asienta la pedagogía. El cuestionamiento de este principio permitiría dar cabida al conflicto en el discurso pedagógico, así como poner en crisis algunas de las bases sobre las que asentamos el análisis de la diversidad y su atención educativa. Nos puede definir la diferencia en lugar de la homogeneidad, lo que implica el cuestionamiento de la norma.

Palabras clave: identidad; diversidad; diferencia; filosofía de la educación. 


\section{SUMMARY}

Multiple definitions about human beings promise to find what is in common in humans and generalize to the whole mankind. This issue is rooted in the principle of identity, and it has a great impact on the mind set where are settled the educational sciences. The challenge to this principle of identity allows making room for conflict in the pedagogical discourse and questions some of the bases that sustain the analysis of diversity and its educational attention. This article aims to reflect why we do define human beings homogenising them, in the place of think them in their differences.

Key words: identity; diversity; difference; philosophy of education.

\section{SOMMAIRE}

Les différentes définitions sur l'être humain tentent d'identifier une fonctionnalité généralisable pour l'ensemble du genre Homo. Cette question découle du principe d'identité, et a des implications fortes pour les conceptions anthropologiques sur lesquelles se fonde la pédagogie. La remise en cause de ce principe permettra d'introduire le conflit dans le discours pédagogique, ainsi que de mettre en crise certains des fondements sur lesquels s'établit l'analyse de la diversité et son attention éducative. Nous pouvons être définis par la différence plutôt que par l'uniformité, et cela implique la mise en question de la norme.

Mots clés: identité; diversité; différence; philosophie de l'éducation.

\section{INTRODUCCIÓN}

A lo largo de la historia se han realizado diferentes definiciones acerca del ser humano. Todas ellas pretenden encontrar lo que de común hay en los seres humanos, es decir, intentan identificar una característica que sea representativa, y generalizarla así para todo el género Homo. Esta cuestión tiene fuertes repercusiones en las concepciones antropológicas sobre las que se asienta la pedagogía en la actualidad. El presente artículo ${ }^{1}$ pretende reflexionar en torno a una pregunta: ¿Y si la característica que nos define no es la que homogeneiza al ser humano sino la que atiende a la diferencia?

Por supuesto que los seres humanos, como otros animales, tienen que comer, beber y dormir. Todos tenemos ciertos rasgos biológicos en común y no puede haber ninguna duda de que compartimos la naturaleza de otros animales. Pero cuando llegas a sus rasgos de comportamiento distintivos, qué diferente es una

1. Agradecemos a Julián Calderón las reflexiones y críticas realizadas acerca de este artículo y a Rafael Calderón su testimonio y enseñanzas. 

DE LA IDENTIDAD DEL SER A LA PEDAGOGÍA DE LA DIFERENCIA

población humana de otra. No solo se diferencian en los idiomas que hablan -tendrás cierta dificultad en hacerte una idea de la gran cantidad de idiomas diferentes que encontrarás-. Difieren en su forma de vestir, en sus adornos, en su forma de cocinar, en sus usos y costumbres, en la organización de sus familias, en sus instituciones sociales, en sus creencias, en sus normas de conducta, en su mentalidad, en casi todo lo que entra dentro de los modos de vida que siguen. Estas diferencias serán tantas y tan variadas que podrías, a menos que estés advertido de lo contrario, tender a ser persuadido de que no todos eran miembros de la misma especie (Adler, 1985, 159, traducción propia).

Desde esta perspectiva, la normalidad dejaría de ser el atributo determinante en la identificación del ser, y pasaría la particularidad a ser el atributo por antonomasia para el entendimiento humano. Se trata de una línea de pensamiento que deja desprovisto de todo significado el proceso de estigmatización por el que la diferencia se vuelve objeto de discriminación. Si es el entorno social el que establece las categorías de las personas que lo conforman, y es la diferencia la esencia del ser, tendremos que ahondar en esta última para repensar la naturaleza humana. Por último, y atendiendo a estos supuestos, carecerían de fundamentación todas aquellas categorías que señalan a las diferencias para justificar la desigualdad humana.

\section{El PROBLEMA DE LA IDENTIDAD EN LA FILOSOFÍA}

En palabras de Heidegger (1969), la pregunta que pretendemos abordar en este artículo se formula en los siguientes términos: «¿Por qué el ser y no más bien la nada?». Históricamente, el tema de la identidad y diferencia ha sido muy relevante. Tanto lo ha sido así que justifica una incursión en los principios básicos de la metafísica que establece Aristóteles, a saber:

1. Principio de identidad.

2. Principio de no contradicción.

3. Principio de causalidad (enunciado por Leibniz como Principio de razón suficiente).

Para nuestro cometido nos interesan especialmente los dos primeros. El principio de identidad afirma que "todo ente es idéntico a sí mismo», expresado en la fórmula: A = A. Este es el primer principio fundamental de la lógica del pensar. El segundo principio añade que esta fórmula no puede ser contradictoria, es decir, que el ente (lo que es, A) no puede ser y no ser al mismo tiempo bajo el mismo aspecto $(\mathrm{A} \neq \mathrm{No} \mathrm{A})$. El tercer principio plantea que todo lo que es debe tener una razón de ser.

Estos principios han tenido un determinante calado en la historia del conocimiento universal, y muy concretamente en la historia de la filosofía. En palabras de Heidegger "lo que expresa el principio de identidad, escuchado desde su tono 
fundamental, es precisamente lo que piensa todo el pensamiento europeo occidental, a saber, que la unidad de la identidad constituye un rasgo fundamental en el ser de lo ente» (Heidegger, 1990, 67). Sin embargo, estos principios van mucho más allá de la filosofía, puesto que, por ejemplo, guardan una inmediata correspondencia con los axiomas matemáticos.

¿Dónde se inician estos principios? La tradición filosófica los asocia a lo expresado por Parménides en el Poema del Ser, del que reproduciremos a continuación un fragmento:



Es decir, Parménides, y toda la línea platónica idealista, identifica realidad e idealidad: pensar $=$ ser. Para Heidegger, en la última frase citada, «lo distinto, pensar y ser, se piensan como lo mismo" (Heidegger, 1990, 69).

Aristóteles (2008) advierte el problema y lo plantea de un modo diferente: hay una correspondencia entre el ser y el pensar, es decir, que los primeros principios del ser se deben corresponder con los primeros principios del pensar. Por tanto, mientras que en el idealismo platónico se identifica ser y pensar, en la línea realista que comienza en Aristóteles esta relación no es de identidad sino de correspondencia. Aristóteles representa el intento por conocer lo físico (el ente móvil) como diferente de lo ideal, pero no alcanza a hacerlo sin elementos lógicos. Para ello, se vale de la abstracción, e introduce la potencia y el acto en el ser, y con ello el movimiento (la física); y plantea que el ser se dice de muchas maneras, intentando distanciarse del pensamiento unívoco: que el ser y el pensar no sean lo mismo. Así pues, a pesar de su pretensión de conocer la realidad, no deja de hacerlo en el logos.

Hasta la modernidad reina el razonamiento inductivo, que consiste en obtener conclusiones generales a partir de datos particulares. Aristóteles define la inducción como «un tránsito de las cosas individuales a los conceptos universales». Es decir, es un razonamiento que va de lo particular a lo universal.

Al llegar la modernidad comienza una ruptura con esta línea de los conceptos universales, y el esfuerzo por hacer de la generalización la herramienta con la que hacer comparecer de un modo preciso toda la realidad, que halla su mayor exponente en Hegel. Esperón lo plantea en los siguientes términos: 
Por otro lado, en la época moderna, el pensar determina la identidad con respecto al ser, manifestándose una nueva concepción de la verdad en cuanto certeza (certeza que tiene el yo-sujeto ante la objetividad del objeto; certeza de la representación). Pienso, luego soy. Dado que fuera del pensamiento nada hay, el ser necesariamente tiene que identificarse con el ser pensamiento. El pensamiento mismo garantiza para sí la certeza de ser. El pensar se presenta idéntico al ser en cuanto conciencia de ser (lo pensado) y autoconciencia de sí (el pensamiento). La época moderna está determinada como Identidad Subjetiva. La identidad es comprendida entre el fundamento y lo fundamentado. Si el rasgo fundamental del ser del ente es ser fundamento; y si el yo, ocupa el lugar del ser como fundamento, entonces, éste se constituye en fundamento de lo real efectivo, es decir, de todo lo ente en general porque satisface la nueva esencia de la verdad decidida en cuanto certeza. Y si su fundamentar (representar claro y distinto) es cierto, entonces, todo representar es verdadero; y si todo representar es verdadero, todo lo que el sujetoyo represente es real (Esperón, 2012, 36).

Como hemos dicho, en la modernidad se rompe con la búsqueda de conceptos universales y con la inducción. Por ejemplo, en Hume (1980) el problema de la inducción reside en que ésta se basa en contingencias (en la experiencia y no en la lógica). Como consecuencia, los conceptos universales se sustituyen en la modernidad por las generalizaciones. Para ello, Hume recurre al hábito:

Cuando hemos descubierto una cierta semejanza entre ideas que en otros aspectos son distintas (por ejemplo, entre las ideas de diversos hombres y de diversos triángulos) empleamos un nombre único (hombre o triángulo) para señalarlas. De este modo se forma en nosotros el hábito de considerar unidas de alguna manera entre sí las ideas designadas con un único nombre; por tanto, el nombre mismo suscitará en nosotros, no una sola de aquellas ideas ni todas, sino el hábito que tenemos de considerarlas juntas y, por consiguiente, una u otra, según la ocasión. La palabra hombre suscitará, por ejemplo, el hábito de considerar a todos los hombres, en cuanto son semejantes entre sí, y nos permitirá evocar la idea de este o aquel individuo particular (Abbagnano, 2000, 320-321).

Más allá del concepto concreto de hábito en Hume, el uso de la generalización (elaborar conceptos que aúnen a otros conceptos) se expande con la modernidad, y con ello se sustituye el razonamiento por inducción por la deducción: de lo general a lo particular, es decir, a la parte. Se crean así dos líneas de pensamiento en la filosofía universal: la línea de la razón (basada en la inducción) y la línea de la generalización (que se asienta en la deducción). La ciencia moderna se decanta claramente por el método hipotético-deductivo.

Sin embargo, la generalización no existe en la realidad. El concepto «animal» no está en la realidad, y a la vez elimina u obvia las diferencias entre el perro y el gato. Por tanto, la generalización, al tratar de buscar lo común en lo diverso, elimina la diferencia. En este sentido, la trisomía 21 no existe si no es, por ejemplo, Calderoniana [de Calderón, el apellido de dos de los autores]. Porque toma el rostro del hermano de dichos autores, que se singulariza en Rafael Calderón. Es decir, que 
no existe la trisomía 21 en términos absolutos. Sin embargo, la ciencia construye la categoría y con ello la generalización: la trisomía 21. Y aunque la trisomía 21 como tal no existe en la realidad, se eliminan las diferencias y se identifica un caso (Rafael Calderón) con nuestro pensamiento (trisomía 21), se iguala la idea con la realidad $^{2}$. Al basarse el pensamiento moderno en el principio de identidad, en el que el pensar es el ser, la representación se torna en verdad gracias al principio de causalidad.

Según Mèlich (2010), mientras sigamos pensando la ética en términos categóricos no hay forma de escapar a lo que él denomina "el escollo del fundamento». Es decir, a aquello que fundamenta al imperativo. Para este autor la ética sólo puede iniciarse desde lo antropológico.

Lo antropológico devuelve sus derechos a la tierra frente a la ilusión de los trasmundos. Y ese devolver los derechos a la tierra significa otorgar la primacía a las situaciones, a los contextos a las preposiciones, a los adverbios, a las relaciones, a los condicionales... así como también a los lazos, a las herencias, a los deseos a las interpretaciones... y cómo no, a los relatos, a las representaciones, a las máscaras, a las transformaciones... Puede verse ahora que lo antropológico es la negación de un punto arquimédico o, dicho de otra forma, es la afirmación de la finitud ${ }^{3}$ (Mèlich, 2010, 316).

Como podemos observar, si para los clásicos la filosofía era el estudio de los primeros principios, para los modernos (y las ciencias modernas) su tarea ha sido el conocimiento desde los primeros principios. Es decir, la modernidad supondría el triunfo de un tipo de conocimiento: el deductivo.

Existen, a pesar de ello, pensadores que no han aceptado los principios fundamentales de la metafísica. Por ejemplo, para Nietzsche la voluntad es preeminente, está por encima de la razón y, por tanto, la actividad está por encima de la presencia. Y más claramente en Heidegger (2001), la preeminencia de la presencia se ha de romper en El Ser y el tiempo a través de un conocimiento del ser en el que pasado, presente y futuro tengan un mismo valor. Para Heidegger, el principio de identidad queda impensado en la historia de la metafísica y constituye el límite del pensamiento filosófico, que se traslada a la filosofía política.

Junto a Heidegger, aunque con diferencias, Derrida pretende continuar el trabajo de liberar a la filosofía del yugo de la metafísica, a través del lenguaje. Por tanto, para liberarse de las cadenas de la superestructura que ha significado la metafísica y su principio de identidad, es necesaria la deconstrucción del lenguaje, desmontar el proceso vivido.

2. Como se ha podido intuir, Rafael Calderón es hermano de dos de los autores y tiene síndrome de Down. Puede conocerse algo más sobre su historia y cómo este tipo de análisis se materializa en la realidad educativa en CALDERÓn ALMENDROS y HABEgGER (2012) y CALDERÓn ALMENDROS (2014).

3. Término empleado por Mèlich $(2010,316-317)$ para describir «el modo de habitar espaciotemporalmente el mundo que habitamos los humanos". 
La deconstrucción del lenguaje es a todos los efectos una tarea compleja, pues no sólo consiste en modificar términos o resguardarnos de los efectos que estos ejercen, sino de ir más allá, de profundizar en el discurso. En el ámbito pedagógico, como señala Skliar (2005), nos hemos preocupado demasiado por esos efectos, por cuidarnos de las palabras, por el empleo del lenguaje "políticamente correcto", pero apenas hemos dedicado tiempo a preguntarnos sobre aquello que dicen esas palabras, la intención con la que se dicen y quién las dice, dando lugar a la confusión. Confusión que genera distinción (siguiendo a Bourdieu, 1998), desigualdad, derivando nuevamente en homogeneización.

Se viene confundiendo digamos trágicamente la/s diferencia/s con los diferentes. Los «diferentes» obedecen a una construcción, una invención, son un reflejo de un largo proceso que podríamos llamar de diferencialismo, esto es, una actitud -sin dudas racista- de separación y de disminución de algunos trazos, de algunas marcas, de algunas identidades en relación con la vasta generalidad de diferencias (Skliar, 2005, 13).

Recapitulemos. Todo comienza en Parménides, cuando al enunciar el principio de identidad que fundamenta la metafísica, identifica el ente (lo que la cosa es) con el pensar (la idea de la cosa). Aristóteles recoge este principio y argumenta que no son lo mismo, sino que hay una correspondencia entre ellos. Aristóteles incorpora el principio de identidad a la física (y por tanto a la ciencia), y a través del principio de causalidad argumenta que el ente ocurre por una razón. Con ello justifica el principio de identidad. Pero a partir de Aristóteles, que sienta las bases de la física y la metafísica (de las ciencias y de la filosofía), se produce un cambio sustancial. Mientras que en la Grecia clásica se estudiaban estos principios a través de la inducción, a partir de la modernidad se asumen estos principios y se inicia el proceso de decantación de la construcción de conocimiento a partir de ellos y a través de la deducción. Por tanto, los principios que constituyen los axiomas de las ciencias y la filosofía quedan al margen de toda discusión crítica, y a partir de ellos se inicia el proceso de construir legítimamente generalizaciones. Esta situación no se cuestiona hasta que Heidegger y algunos filósofos postmodernos, como Derrida, retoman de nuevo el análisis del principio de identidad, que según ellos ha condicionado el desarrollo de la metafísica. Básicamente defienden que la identidad niega la diferencia: si pensar y ser son lo mismo se anula el conflicto y se elimina la diferencia.

De acuerdo con las ideas esgrimidas hasta ahora, las definiciones realizadas acerca del ser humano se han basado en el principio de identidad. Por tanto, todas eliminan la diferencia: el ser humano como animal o ser racional (Gehlen, 1993) o que tiene logos (Aristóteles, citado por Choza, 1988, 133), como animal simbólico (Cassirer, 2005, 40), como ser técnico (Carbonell y Mosquera, 2000, 179), como bomo oeconomicus (Weber, 2008, 468), como el ser cultural por naturaleza y natural por la cultura (Gehlen, 1993, 15), como ser pulsional (Freud, citado por Gehlen, 1993), como dualidad alma-cuerpo (Platón, citado por Stevenson, 1974, 
45-47) y como animal o ser social (Aristóteles, 2003, 73; Platón, 2004), como centro superior a la antítesis del organismo y el medio (Scheler, 1938, 60), como animal de realidades y animal personal (Zubiri, 1980), como homo faber, ser productivo o derivado de las formas de trabajo (Marx, 2004; James, Pierce, Schiller y Dewey, citado por Scheler, 1938, 101), como ser individual y libre (Kierkegaard, 1979), como ser dominado por los impulsos de poderío y prevalecimiento (Maquiavelo y Hobbes, citado por Scheler, 1938, 102), como ser que habita el lenguaje (Heidegger, 2004), etc.

Todas estas definiciones son generalizaciones que buscan la característica común a todos los seres humanos, pero estos conceptos son ideas que son insuficientes. Al aferrarnos a la deducción, el ente queda a expensas de la razón. Y esto, en terrenos del conocimiento social y humano, esconde una especial complejidad.

Si el ente es el ser humano, el problema, como decimos, es aún mayor. Siguiendo a Heidegger $(1990,77)$, el hombre como animal racional de la modernidad "llegó a convertirse en sujeto para su objeto». Es en la mismidad en la que Heidegger encuentra el nuevo camino de la interpretación, "el salto» que nos permite cuestionar el principio de identidad, en la correspondencia entre el ser y el hombre a través del principio de la transpropiación: el hombre es propiamente la relación de correspondencia, la mutua pertenencia de identidad y diferencia.

El pensar necesitó más de dos mil años para comprender propiamente una relación tan fácil como la mediación en el interior de la identidad. ¿Acaso podemos opinar nosotros que la entrada con el pensamiento en el origen de la esencia de la identidad pueda llegar a realizarse algún día? Justamente porque tal entrada necesita un salto, precisa su tiempo, el tiempo del pensar, que es diferente al del calcular, que hoy tira en todo lugar de modo violento de nuestro pensar. Hoy en día, la máquina del pensar calcula en un segundo miles de relaciones: a pesar de su utilidad técnica están privadas de esencia.

De cualquier modo que intentemos pensar y pensemos lo que pensemos, pensarnos en el campo de la tradición. Esta prevalece cuándo nos libera del pensar en lo pasado para pensar por adelantado, lo que ya no es ningún planear.

Sólo cuando nos volvemos con el pensar hacia lo ya pensado, estamos al servicio de lo por pensar (Heidegger, 1990, 95-97).

Lo que añade Heidegger en el Ser es precisamente lo que da título a su principal obra: El Ser y el tiempo (Heidegger, 2001). El tiempo ofrece movimiento al Ser, rompiendo con ello el carácter estático del principio de identidad. De esta manera puede distanciarse de la extendida comprensión de la mismidad: no solo reconoce la diferencia del pensar y de lo ente, sino que el tiempo opera la diferencia en el sujeto que piensa. Si esto tiene una gran relevancia en el análisis ontológico, cuánto más en el análisis antropológico que pretendemos hacer: los seres humanos no son iguales entre sí, ni siquiera una persona es igual a sí misma, puesto que tiene biografía. Nunca será idéntico. Por ello, la postmodernidad plantea que el pensamiento violenta al ser. Tanto Heidegger como Derrida plantean la salida a la determinación del conocimiento filosófico con la historia, produciendo una 
violencia epistémica o violencia interpretativa, que permita revisar la tradición y desmontarla: es lo que Derrida llamará deconstrucción (procedente del concepto Dekonstruktion generado por Heidegger), que realiza a través de la descomposición de la estructura del lenguaje. Es lo que se ha venido a llamar filosofía de la diferencia.

\section{El PROBLEMA DE LA IDENTIDAD COMO REALIDAD SOCIAL}

Foucault, en la sociología, construye una línea con la que pretende cuestionar la tradición y la racionalidad moderna para romper sus límites, permitiendo con ello nuevas formas de ideas. De hecho crea una metodología para indagar en «las cosas dichas»: la arqueología del saber (Foucault, 1997), que representa la búsqueda de la historia viva en el presente. Para él la historia muestra claves del presente, pero necesita un tratamiento distinto del tiempo, que no puede ser lineal. A través de los documentos, Foucault realiza una reconstrucción de las relaciones que se establecieron a partir de ellos.

Esperón (2012) elabora un formidable análisis en el que muestra (basándose en Espósito, Heidegger y Foucault) cómo el problema de la identidad planteado en estas páginas acaba por convertirse en realidad política y social. El límite de la metafísica (el principio de identidad) reduce la política a su propio orden categorial, y piensa el conflicto desde el orden posible, sustituyendo el conflicto por orden. Tal como expone el autor, «[d]e esto se desprende que la realidad de la política es el conflicto, la diferencia, la multiplicidad, pero esto es precisamente lo que no entra en los esquemas representativos de la filosofía política» (Esperón, 2012, 33). Es decir, se reduce lo múltiple a lo uno, como veníamos viendo al hablar de la generalización.

Como consecuencia, en la modernidad surge la burocracia (Weber, 2008), que conlleva según Mèlich (2003) «un incesante proceso de anonimización»: el ser humano pasa a ser considerado objeto y su vida se convierte en expediente. También recalca Mèlich el «imperio de la racionalidad instrumental» en la modernidad, en el cual «lo que es [el ser humano] se convierte en cosa» a través de la producción tecnológica, que requiere el anonimato, la uniformidad y el estereotipo. Por tanto, como consecuencia de ensalzar al sujeto [cogito] a través del principio de identidad [pensar es ser], se elimina la diferencia, y los hombres más allá del yo-sujeto se convierten en objetos a través del lenguaje, la burocracia y la racionalidad instrumental. Ha desaparecido el conflicto, la diferencia, y el sujeto pasa a formar parte de la representación del orden institucional, despolitizándose.

Estas instituciones, según Foucault (2002), buscan el mismo objetivo: normalizar. Es lo que denominó sociedades disciplinarias:

Foucault sostiene que el carácter distintivo y decisivo del siglo XX es el pasaje del ejercicio del poder basado en el principio de soberanía (donde el carácter jurídico de la ley ejerce el poder como instancia ordenadora del pueblo sujeto 
político) a otra forma del ejercicio del poder denominado biopolítica (basada en el principio de normalización y desplegada a través de dispositivos de control y administración de la vida que produce y regula las sociedades -sujeto biológico-). A este tipo de sociedades las denominó sociedades disciplinarias o sociedades de encierro. En ellas, el ejercicio del poder produce saberes que a su vez retroalimenta y perfecciona el ejercicio del poder, que circula independientemente de los sujetos pero, a su vez, sujetándolos. En estas sociedades, las instituciones de encierro controlan y moldean las subjetividades con el fin de prevenir y adelantarse a los posibles delitos. Estas instituciones son concebidas sobre la base de la identidad (comprendida como principio de normalización de conductas). De este modo, sólo somos sujetos políticos en la medida en que somos sujetados efectivamente. De allí el deseo de querer ser sujetado, pues sólo de ese modo, se es un actor social reconocido institucionalmente (Esperón, 2012, 38).

De esta forma se reduce lo múltiple a lo uno porque, como mantiene Foucault (Esperón, 2012), quien no se adecua a la identidad normalizadora institucional es excluido, no es reconocido como sujeto; se excluye de una institución para una mayor integración y normalización en la siguiente; las personas no institucionalizables quedan excluidas, y son causa de integración del resto. Así todos son productivos. Sin embargo, lo que Derrida $(1968,25)$ llama «innombrable» por no poder ser incluido dentro de la lógica filosófica, lo que «no se presta al juego de la oposición ni de su lógica" tiene la capacidad de dejar sin efecto al orden (Skliar, 2007, 44).

La normalización ha ejercido un efecto demoledor en la construcción de nuestro pensamiento y en nuestra manera de entender el mundo. Tanto es así, que a menudo se ha empleado la diferencia como justificación de la desigualdad, asociando el término a lo "no deseable», a aquello conducente a la discriminación, marginación, exclusión, etc. En el mundo no existen esencias (siguiendo a Nietszche, 1873), somos nosotros quienes atribuimos identidad, quienes ponemos atributos y quienes finalmente creamos el estigma (Goffman, 1963). En definitiva, es la propia especie humana quien por intentar hacer orden del caos ha desnaturalizado su propia esencia en aras de la debilidad que conlleva el enfrentamiento a lo diferente, a lo desconocido, a lo que aún no ha sido normalizado.

\section{DE LA IDENTIDAd A LA PEDAGOGía DE LA DifERENCIA}

Visto un león, están vistos todos. Y vista una oveja, todas. Pero visto un hombre, no está visto sino uno, y aun ése no bien conocido (Gracián, 1967, 622).

Cuando nacemos lo hacemos en un mundo que nos viene dado, con unos hábitos de conducta, valores, signos, símbolos... que son heredados. Según Mèlich (2010) todo ello forma parte de una gramática de marcos normativos que nos impone obligaciones y a la cual necesitamos someternos para sobrevivir en nuestro mundo. Ahora bien, el ser humano es capaz de posicionarse frente a ese mundo, y romper con esa gramática o identidad heredada aunque sólo sea por un momento. En esa transgresión es donde entra en juego la ética, y lo que nos ofrece sentido. 
Todo lo argumentado nos debe hacer pensar la necesidad de generar esa violencia epistémica que cuestione la identidad. En este sentido, la pedagogía debería dejar de centrarse en el ¿qué somos?, para sustentarse en una antropología narrativa que se preocupe por la singularidad del individuo y por sus posibilidades de poner en tela de juicio el orden establecido por nuestro sistema cultural e institucional (Mèlich, 2008).

Ello implica la deconstrucción del conocimiento pedagógico que hemos ido montando a partir de clasificaciones, estándares, etiquetados, catálogos, baterías, etc. Deconstruir las exclusiones que ha generado el orden actual. Esta ruptura en el ámbito de la antropología es una aceptación de la diferencia por encima de la identidad, del conflicto por encima del orden. Lo común a todos los seres humanos es precisamente lo diferente: no hay un ser humano igual a otro, no hay un ser humano que no cambie. Cada hombre o mujer tiene una vida, una biografía. El ser humano, por tanto, es diferente de sí mismo y de los demás. Es la libertad, la apertura, la inconclusión la que nos permite la diferencia; es a través del poder que se niega. Por la libertad somos diferentes de nosotros mismos y de los demás. A través del control se nos pretende iguales y estáticos.

La identidad "que hemos heredado funciona a modo de "bloqueo", neutralizando determinadas posibilidades de ser -de intimidad, en el caso que nos ocupa-, y esta neutralización es, además, sumamente sutil porque no se sitúa en el exterior de cada uno de nosotros sino en el interior» (Mèlich, 2010, 327).

Por ello, una pedagogía de las diferencias (Skliar, 2007; Skliar y Téllez, 2008) requiere un nuevo posicionamiento ante la realidad y las relaciones educativas. Un posicionamiento cargado de incertidumbre, en tanto que debe violentar algunas de las bases sobre las que hemos ido construyendo el conocimiento pedagógico, las relaciones educativas y el sentido mismo de instituciones como la escolar. Porque la tradición privilegia un conocimiento pedagógico restringido a la tematización y a la normalidad, y no hay hecho pedagógico si no se problematizan las relaciones en lugar de cuestionar al otro (Skliar, 2008, 11-12). En este cometido, las teorías de la resistencia y la pedagogía de los límites (o de la frontera) permiten analizar, desafiar y transformar las representaciones y prácticas educativas que nombran, marginan y definen la diferencia como «el otro devaluado", cuestionando las relaciones de poder que sustentan esta "colonización de las diferencias» (Giroux, 1994).

Una pedagogía de la diferencia necesita volver nuestra mirada hacia los procesos educativos, personas y colectivos que son sistemáticamente olvidados por la tradición. Constatar con Foucault que la racionalidad occidental se ha construido por una serie de exclusiones: la locura, la enfermedad, la delincuencia..., de modo que es necesario volver a pensar lo impensado. Descomponer el lenguaje y la realidad social para observar cómo se privilegia un elemento central a la vez que se dejan al margen otros. Contemplar cómo la norma elimina sistemáticamente cualquier otra forma, y transgredirla. "[E]scribir de nuevo la diferencia mediante el proceso de atravesar las fronteras culturales que ofrecen narrativas, lenguajes y 
experiencias que proporcionan un recurso para repensar la relación entre el centro y los márgenes del poder, así como entre ellos mismos y los demás» (Giroux, 1997, 206).

Atravesar fronteras, e incluso vivir en ellas. Deconstruir, al fin y al cabo, todo aquello que nos separa y excluye. Una tarea que, según Bolívar (1990, 189), no es «de destrucción o demolición de las oposiciones clásicas para quedarse en un monismo o en un nuevo centro, sino situarnos en el límite del discurso filosófico, pero dentro de él, para intentar desbordarlo». Se trata de verlos desde su Otro innombrable.

En el terreno educativo este cuestionamiento ha de hacerse a través de la deconstrucción del conocimiento pedagógico generado a partir de clasificaciones, estándares, etiquetados, catálogos, baterías, etc. Para Derrida (2005), la deconstrucción se centra en mostrar y transformar las relaciones jerárquicas que conforman la vida cotidiana. Deconstruir implica cuestionar lo que nos resulta natural y provoca una generalización de la racionalidad. Por tanto, como educadores tenemos que volver a mirar lo mismo, conscientes ahora de que «estamos invadidos de saberes y discursos que patologizan, culpabilizan y capturan al otro, trazando entre él y nosotros una rígida frontera que no permite comprenderle, conocerle ni adivinarle» (Pérez de Lara, 2001, 296). Una mirada que requiere la deconstrucción de las exclusiones que ha generado el orden actual, aceptando la diferencia por encima de la identidad, el conflicto por encima del orden. La inconclusión del ser humano es la que nos permite la diferencia; es a través del poder normalizador que se niega. Por la libertad somos diferentes de nosotros mismos (en tanto que cambiamos con el tiempo) y de los demás. A través del control se nos pretende iguales y estáticos.

Sin embargo, lo que no entra en la lógica normalizadora tiene el poder de dejar sin efecto al orden que excluye la diferencia (Skliar, 2007) y elimina el conflicto. En este cometido, las teorías de la resistencia y la pedagogía de los límites (o de la frontera) permiten localizar, analizar, desafiar y transformar las representaciones y prácticas educativas que devalúan al otro y castigan las diferencias, creándose con ello una nueva narrativa que desafía el orden previo y que requiere la diferencia. Para ello hay que resituar el debate pedagógico en las arenas de la ética y la justicia.

La pedagogía de los límites muestra cómo el poder se introduce en el sujeto, en la cultura y en el mundo físico. Las fronteras dividen, clasifican y ordenan respecto al poder. Incluyen y excluyen. Constituyen fortalezas que aseguran el statu $q u o$, pero son a la vez los límites del ideario que las sostiene, las fisuras del sistema que oprime, que pueden ser cuestionadas a través de la diferencia. Al reconocer las fronteras como inestables y precarias, comprendemos que se pueden cruzar. Las fronteras, que son los límites de la lógica que niega la diferencia, pueden habitarse y cuestionar las categorías que las sustentan. Y al hacerlo, se generan nuevas cartografías vitales, sociales y culturales, ya que permiten ampliar las posibilidades de conocer la realidad y de construcción creativa. Cuando esto ocurre, se está en condiciones de construir una identidad a través de la interpretación (Ruiz Román, 
Calderón Almendros y Torres, 2011) y, con ello, dibujar un nuevo mapa de las relaciones educativas.

Es posible entonces habitar las fronteras y los límites, lo que en la pedagogía podría significar la ruptura con dicotomías excluyentes. Una empresa no exenta de problemas como plantean Planella y Pie (2012), por las resistencias de la tradición y sus aparatos de control. En cualquier caso, uno de nosotros (Calderón Almendros, 2014) ha apuntado algunas de esas fronteras pedagógicas que hay que diluir, deconstruir, superar o habitar. Como las fronteras entre la biología y la cultura; la frontera de la humanidad que en la actualidad excluye a las personas con discapacidad; la frontera de la normalidad que bajo su tiranía (el de la educación como molde según Esteve, 2010) impide que se ensanche y revitalice nuestra humanidad; la frontera de la educación especial y las áreas de conocimiento, que impiden análisis más holísticos y menos excluyentes; la frontera de la inteligencia, que reduce hasta lo impensable la naturaleza multidimensional del ser humano; la frontera del lenguaje, que se podría superar educativamente habitando otros logos como la música, el arte, las emociones...; la frontera entre el aprendizaje y la participación que hizo ver Doyle (1977), porque no caben la una sin la otra, lo que impediría las segregaciones obscenas que las escuelas siguen impulsando y legitimando; la frontera entre la teoría y la práctica, que reduce el compromiso y la capacidad de transformación de la educación y la investigación educativa; la frontera entre lo individual y lo social, que no existen por separado sino en la construcción del sujeto; y la frontera de la realidad y los sueños, que responde a la naturaleza narrativa de la realidad (Brunner, 1991). La educación ha de responder a esta realidad para acompañar en lo que MacIntyre (1987) llama la vida como búsqueda. La vida es narración, es apertura. Por tanto, no es solo realidad, no es solo acto; es sueño, es potencia. Por eso, y a pesar de los discursos paralizantes que proliferan en estos días, la educación es, por encima de cualquier cosa, esperanza.

Un proceso educativo de esta índole permite invertir esas posiciones, creándose con ello un nuevo texto, una nueva narrativa que desafía el orden previo y que requiere la diferencia. Necesitamos desenfocar nuestras prácticas e investigaciones educativas para volver a enfocar en lo periférico ${ }^{4}$, restaurando con ello la capacidad de decidir de la comunidad, empoderando a las personas y colectivos oprimidos $^{5}$, y actuando en las estructuras narrativas y de responsabilidad moral del grupo (Denzin, 2008, 196). Rescatar la acción educativa que el positivismo y su concepción de la identidad ha "cosificado» convirtiéndola en una intervención

4. Como afirma SHuTtera (2006, 103), "Derrida suele ser asociado a pensadores como Foucault, Deleuze, Lyotard, entre otros. Sin embargo, sería difícil explícitamente reducirlos a ser "pensadores de la diferencia", sin más, dado que todos ellos son fundamentalmente reticentes a ocupar un lugar dentro de un estante filosófico o una "corriente" homogénea dentro de la historia de las ideas".

5. Algunas de las investigaciones educativas que hemos realizado en este sentido pueden consultarse en los siguientes trabajos: Ruiz Román, CALderón Almendros y Torres (2011), Calderón Almendros (2011), Sepúlveda, Calderón Almendros y Torres (2012), Calderón Almendros y HabegGer (2012) y CALDERÓN ALMENDROS (2014). 
supuestamente controlada en aras de la eficacia (Ortega, 2004, 7), para resituar el debate pedagógico en las arenas de la ética y la justicia. Y ese nuevo enfoque ha de ser cualitativamente distinto, ya que ha de permitir el desorden que cuestione los regímenes de verdad. Solo así podríamos conseguir que las personas y colectivos excluidos puedan ser incluidos sin ser fagocitados.

\section{REFERENCIAS BIBLIOGRÁFICAS}

Abbagnano, N. (2000) Historia de la filosofía, vol. II. Barcelona, Hora.

Adler, M. J. (1985) Ten philosophical mistakes. New York, MacMillian Publishing Company. ARISTÓteles (2001) Ética a Nicómaco. Madrid, Alianza Editorial.

ARistóteles (2008) Metafísica. Madrid, Alianza Editorial.

Aristóteles (2003) Política. Santa Fe, El Cid Editor.

Bolívar, A. (1985) El estructuralismo: de Levi Strauss a Derrida. Madrid, Cincel.

Bourdieu, P. (1998) La distinción: Criterio y bases sociales del gusto. Madrid, Taurus.

Brunner, J. (1991) Actos de significado. Más allá de la revolución cognitiva. Madrid, Alianza Editorial.

Calderón Almendros, I. (2011) Breaking away to find a way: poverty and school failure in a Spanish adolescent life-history. British Journal of Sociology of Education, 32 (5), 745-762.

Calderón Almendros, I. (2014) Educación y esperanza en las fronteras de la discapacidad. Madrid, CERMI.

Calderón Almendros, I. y Habegger Lardoeyt, S. (2012) Educación, hándicap e inclusión. Una lucha familiar contra una escuela excluyente. Granada, Octaedro Andalucía.

Carbonell, E. y Mosquera, M. (2000) Las Claves del pasado. La llave del futuro. Tarragona, Arola Editors.

Cassirer, E. (2005) Las ciencias de la cultura. México D. F., Fondo de Cultura Económica.

Choza, J. (1988) Manual de antropología filosófica. Madrid, Rialp.

Denzin, N. K. (2008) La política y la ética de la representación pedagógica: hacia una pedagogía de la esperanza, en Mclaren, P. y Kincheloe, J. L. (eds.) Pedagogía crítica: de qué hablamos, dónde estamos. Barcelona, Graó, 181-200.

Derrida, J. (1968) La diferencia/[Différance]. Chile, Escuela de Filosofía Universidad ARcis. Consultado el 12 de octubre de 2012. http://www.uruguaypiensa.org.uy/imgnoticias/590.pdf.

DerridA, J. (2005) De la gramatología. Buenos Aires, Siglo XXI.

DoyLe, W. (1977) Learning the classroom environment: an ecological analysis. Journal of Teacher Education, 28 (6), 51-55.

Esperón, J. P. (2012) El principio de identidad como principio de exclusión. Identidades, 2 (2), 30-41. Consultado el 12 de octubre de 2012. http://iidentidadess.files.wordpress. com/2012/06/identidades-2-2-20122.pdf.

Foucault, M. (1997) La arqueología del saber. México: Siglo XXI.

Foucault, M. (2002) Vigilar y castigar. Nacimiento de la prisión. Buenos Aires, Siglo xxI.

GeHlen, A. (1993) Antropología filosófica. Del encuentro y descubrimiento del hombre por sí mismo. Barcelona, Paidós.

Giroux, H. A. (1994) Democracy and the Discourse of Cultural Difference: Towards a Politics of Border Pedagogy. British Journal of Sociology of Education, 12 (4), 501-519. 
Giroux, H. A. (1997) Cruzando limites. Trabajadores culturales y politicas educativas. Barcelona, Paidós.

Goffman, E. (1963) Estigma. La identidad deteriorada. Buenos Aires, Amorrortu.

Gracián, B. (1967) El golfo cortesano: Obras completas. Madrid, Aguilar.

Heidegger, M. (1969) Introducción a la metafísica. Buenos Aires, Editorial Nova.

Heidegger, M. (1990) Identidad y diferencia. Barcelona, Anthropos Editorial.

Heidegger, M. (2001) El ser y el tiempo. México D. F., Fondo de Cultura Económica.

Heidegger, M. (2004) Carta sobre el humanismo. Madrid: Alianza Editorial.

Hume, D. (1980) Investigación sobre el Conocimiento Humano. Madrid: Alianza.

KierkegaARD, S. (1979) El concepto de la angustia. Madrid, Espasa-Calpe.

Kirk, G. S.; Raven, J. E. y Schofield, M. (2003) Los filósofos presocráticos. Madrid, Gredos.

MacinTYRe, A. (1987) Tras la virtud. Barcelona, Crítica.

Marx, K. (2004) Manuscritos económico-filosóficos de 1844. Buenos Aires, Ediciones Colihue SRL.

MÈLICH, J. C. (2003) Memoria y esperanza. Coimbra, Associação de Professores de Filosofia. Consultado el 12 de octubre de 2012. http://www.apfilosofia.org/documentos/pdf/ MelichSant.pdf.

MÈLICH, J. C. (2008) Antropología narrativa y Educación. Teoría de la Educación, 20, 101-124

MÈLICH, J. C. (2010) Poética de lo íntimo (Sobre Ética y Antropología). Ars Brevis, 16, 314-331.

Nietzsche, F. (1873) Sobre verdad y mentira en sentido extramoral. Trad. por S. Hoyo Hernández. Consultado el 3 de noviembre de 2014. http://www.lacavernadeplaton.com/ articulosbis/verdadymentira.pdf.

Ortega, P. (2004) La Educación Moral como Pedagogía de la Alteridad. Revista Española de Pedagogía, 227, 5-30.

PéreZ De LARA, N. (2001) Identidad, diferencia y diversidad: mantener viva la pregunta, en Larrosa, J. y Skliar, C. (eds.) Habitantes de Babel. Políticas y poéticas de la diferencia. Buenos Aires, Aguazul, 291-316.

Planella, J. y Pie, A. (2012) Pedagoqueer: Resistencias y subversiones educativas. Educación XXI, 15 (1), 265-283.

Platón (2004) Protágoras. Santa Fe, El Cid Editor.

Ruiz Román, C.; Calderón Almendros, I. y Torres Moya, F. J. (2011) Construir la identidad en los márgenes de la globalización: educación, participación y aprendizaje. Cultura y Educación, 23 (4), 589-599.

SCHeler, M. (1938) El puesto del hombre en el cosmos. Buenos Aires, Editorial Losada.

Sepúlveda Ruiz, M. P.; Calderón Almendros, I. y Torres Moya, F. J. (2012) De lo individual a lo estructural. La investigación-acción participativa como estrategia educativa para la transformación personal y social en un centro de intervención con menores infractores. Revista de Educación, 359, 456-480.

Shuttera, A. S. (2006) Derrida: la estructura desplazada y el problema de la différance. Liminar. Estudios Sociales y Humanísticos, 4 (2), 93-108. Consultado el 12 de octubre de 2012. http://redalyc.uaemex.mx/pdf/745/74540208.pdf.

Skliar, C. (2005) Poner en tela de juicio la normalidad, no la anormalidad. Políticas y falta de políticas en relación con las diferencias en educación. Revista Educación y Pedagogía, Medellín, Universidad de Antioquía, Facultad de Educación, 17 (41), 11-22.

SKliar, C. (2007) ¿Y si el otro no estuviera abí? Notas para una pedagogía (improbable) de la diferencia. Buenos Aires, Miño y Dávila. 
SkLIAR, C. (2008) ¿Incluir las diferencias? Sobre un problema mal planteado y una realidad insoportable. Orientación y Sociedad, 8, 1-17. Consultado el 18 de octubre de 2012. http://www.scielo.org.ar/pdf/orisoc/v8/v8a02.pdf.

Skliar, C. y Téllez, M. (2008) Conmover la educación. Ensayos para una pedagogía de la diferencia. Buenos Aires, Noveduc.

STEVENSON, L. (1974) Siete teorías de la naturaleza humana. Madrid, Cátedra.

Weber, M. (2008) Economía y sociedad. Esbozo de una sociología comprensiva. México D. F., Fondo de Cultura Económica.

ZuBIRI, X. (1980) Inteligencia sentiente. Madrid, Alianza. 